\title{
IGF-1 levels correlate with T3 status in Chronic Heart Failure outpatients: preliminary data
}

${ }^{1}$ V. Triggiani, ${ }^{2}$ M. lacoviello, ${ }^{2}$ M. Leone, ${ }^{1}$ V.A. Giagulli, ${ }^{1}$ E. Guastamacchia, ${ }^{3}$ A. Salzano, ${ }^{3}$ A. Bobbio, ${ }^{4}$ M. Arcopinto, ${ }^{3} \mathrm{~A}$. Cittadini, on behalf of TOSCA (Trattamento Ormonale nello Scompenso CArdiaco) investigators

${ }^{1}$ Endocrinology and Metabolic Diseases, Interdisciplinary Department of Medicine, University of Bari, Bari, Italy; ${ }^{2}$ Cardiology Unit and Cardiothoracic Department, Policlinico Consorziale University Hospital, Bari, Italy; ${ }^{3}$ Department of Medical Translational Sciences, Federico II University, Naples, Italy; ${ }^{4}$ Department of Cardiac Surgery, IRCCS Policlinico S. Donato Milanese, Milan, Italy

\section{OBJECTIVES}

Increasing evidence indicates that a variety of hormones may be down-regulated in CHF patients. Impaired activity of the GH/IGF-1 axis in CHF (low IGF-1 levels, GH deficiency, and $\mathrm{GH}$ resistance) has been described by several studies and is associated with poor clinical status and outcome. Multiple cross-sectional studies, moreover, demonstrate that a decrease in serum T3 in CHF patients is correlated to the severity of the heart disease as assessed by the NYHA classification. Aim of this study was to evaluate a possible correlation between IGF-1 levels and T3 status in a cohort of CHF outpatients.

\section{METHODS}

Forty-eight consecutive CHF outpatients (79\% males; age $61 \pm 13$ years; BMI $29 \pm 5 \mathrm{Kg} / \mathrm{m}^{2}$; NYHA Class $2.3 \pm 0.6 ; 47 \%$ with ischemic disease), in stable clinical conditions from at least 30 days, in conventional electrical and medical therapy (87\% taking ACEinhibitors or angiotensin receptor blockers, $96 \%$ beta blockers, $96 \%$ diuretics, $72 \%$ anti-aldosterone drugs, $13 \%$ digitalis, $14 \%$ nitrates), were enrolled in the study. They were submitted to physical examination, electrocardiography and echocardiography. Blood samples were drawn to assess renal function, $\mathrm{Na}+$, hemoglobin, NT-proBNPs, fT3, fT4, TSH, IGF-1, testosterone, DHEA and insulin levels.

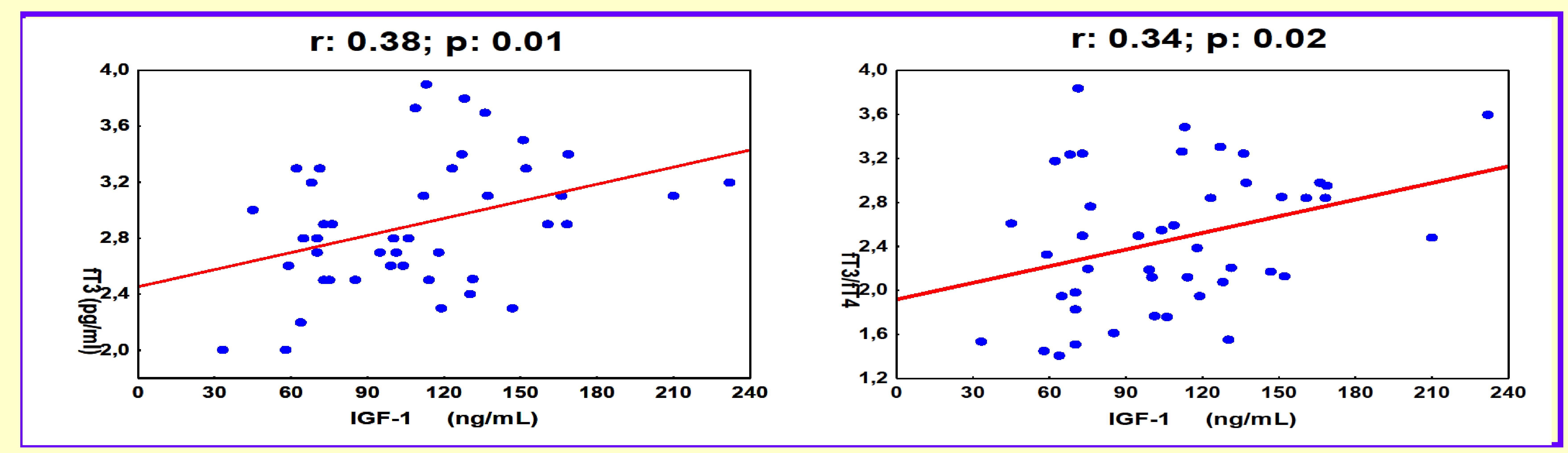

Fig. 1 IGF-1 levels directly correlate to both fT3 levels and fT3/fT4 ratio

\section{RESULTS}

At univariate analysis, IGF-1 showed a direct correlation with FT3 and the same was found between IGF-1 levels and fT3/fT4 ratio, whereas no correlation was found between IGF-1 and the other measures. Furthermore, at multivariate analysis, including also NHYA class, fT3 was the only independent predictor of IGF-1 levels.

\section{CONCLUSIONS}

Impaired IGF-1 and fT3 status may both represent a derangement strictly correlated to the severity of the clinical condition in CHF. 